\title{
THE AXIS OF ROTATION OF THE ANKLE JOINT
}

\author{
ARNE LUNDBERG, OLA K. SVENSSON, GUNNAR NÉMETH, GÖRAN SELVIK
}

From the Karolinska Hospital, Stockholm and Lund University, Sweden

\begin{abstract}
The axis of the talo-crural joint was analysed by roentgen stereophotogrammetry in eight healthy volunteers. Examinations were performed at $10^{\circ}$ increments of flexion and pronation/supination of the foot as well as medial and lateral rotation of the leg. Results indicate that the talo-crural joint axis changes continuously throughout the range of movement. In dorsiflexion it tended to be oblique downward and laterally. In rotation of the leg, the axis took varying inclinations between horizontal and vertical. All axes in each subject lay close to the midpoint of a line between the tips of the malleoli.

Our study indicates that the talo-crural joint axis may alter considerably during the arc of motion and differ significantly between individuals. This prompts caution in the use of hinge axes in orthoses and prostheses for the ankle.
\end{abstract}

Kinematics of the joints of the ankle and foot is a field of biomechanical research in which methods of reliable and accurate measurement in vivo are lacking. Of the many joints of the region, the talo-crural joint is the only one to show uniformity in its kinematic properties between individuals.

There is, however, some controversy concerning the axis of this joint, particularly the clinical significance of a shift close to the neutral position between plantarflexion and dorsiflexion observed by, among others, Barnett and Napier (1952) and Hicks (1953). These investigators found that the joint axis in dorsiflexion ran from a medial and slightly proximal position in an oblique direction downward and laterally. In plantarflexion the axis ran from a medial and inferior position in a lateral and proximal direction. In Hicks' study, which has been accepted by later investigators (Allard, Thiry and Duhaime 1985) the axes for dorsiflexion and plantarflexion are shown to differ, with approximately 20 to $30^{\circ}$ between the two axes. Nothing is said about how the change-over between these two axes occurs, but the description of only two axes during continuous motion indicates a rather abrupt change near the neutral position. This observation was not confirmed by Inman (1976) in a later study which, despite considerable

A. Lundberg, MD, Lecturer

G. Németh, MD, PhD, Lecturer

Department of Orthopaedic Surgery, Karolinska Hospital, S-104 01, Stockholm, Sweden.

O. K. Svensson, MD, PhD, Lecturer

Department of Anatomy, Karolinska Institute, Stockholm, Sweden.

G. Selvik, MD, PhD, Associate Professor

Department of Anatomy, Lund University, Lund, Sweden.

Correspondence should be sent to Dr A. Lundberg.

(C) 1989 British Editorial Society of Bone and Joint Surgery 0301-620X/89/1031 \$2.00

J Bone Joint Surg [Br] 1989;71-B:94-9.
Table I. Age and sex of the eight normal subjects

\begin{tabular}{lll}
\hline Subject & $\begin{array}{l}\text { Age in } \\
\text { years }\end{array}$ & Sex \\
\hline 1 & 33 & M \\
2 & 31 & M \\
3 & 34 & F \\
4 & 38 & M \\
5 & 26 & F \\
6 & 29 & M \\
7 & 32 & M \\
8 & 33 & M \\
\hline
\end{tabular}

individual variations, tended to indicate a rather simple, hinge-like, action of the joint in dorsiflexion and plantarflexion.

Studies of movement in the ankle other than plantarflexion and dorsiflexion have generally dealt with instability rather than physiological movement (Laurin and Mathieu 1975; Stormont et al. 1985; Larsen 1986). Close (1956), however, mentioned rotation about a vertical axis in the ankle joint during walking, and McCullough and Burge (1980), in one of the few investigations of such movement, analysed the range of movement about the vertical axis in anatomical preparations. The results indicated a range of horizontal rotation of approximately $18^{\circ}$ at $50 \mathrm{~kg}$ joint load. Van Langelaan (1983) made a roentgen stereophotogrammetric analysis of joint movement and discrete joint helical axes using cadaveric specimens. When the leg was moved from medial to lateral rotation, the joint axis was amost horizontal, except for the first phase (initial movement 


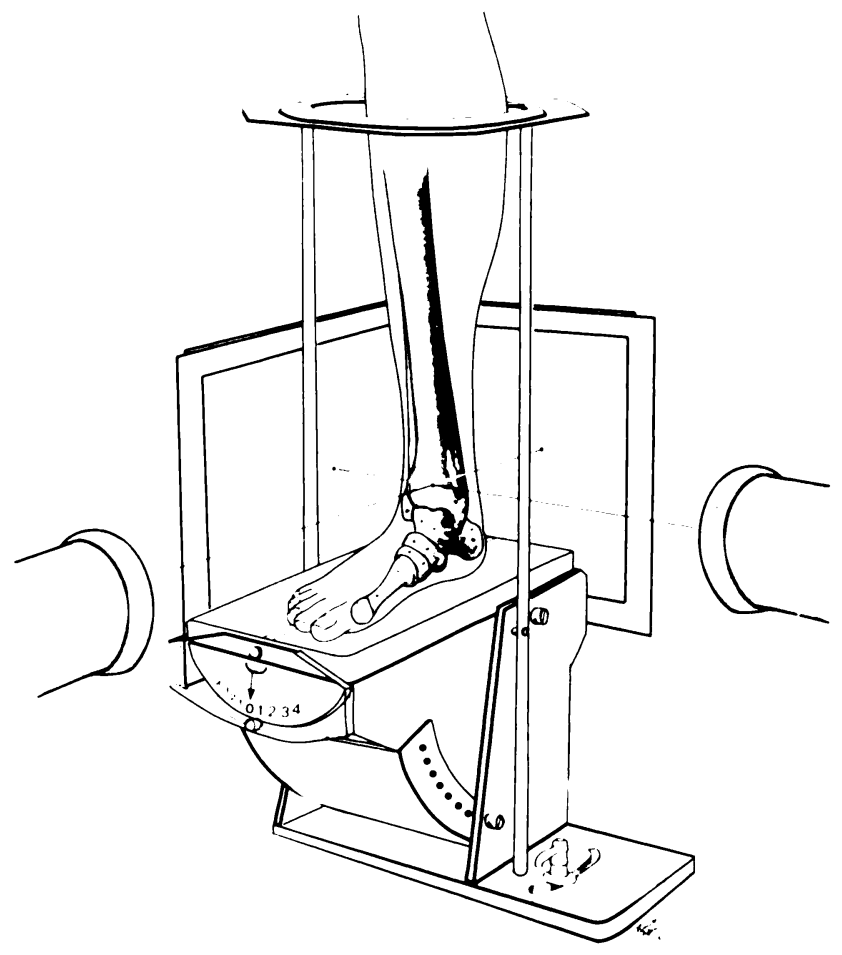

Fig. 1

Diagram to show the apparatus with a leg in position and $x$-ray tubes placed for anteroposterior and lateral exposures.

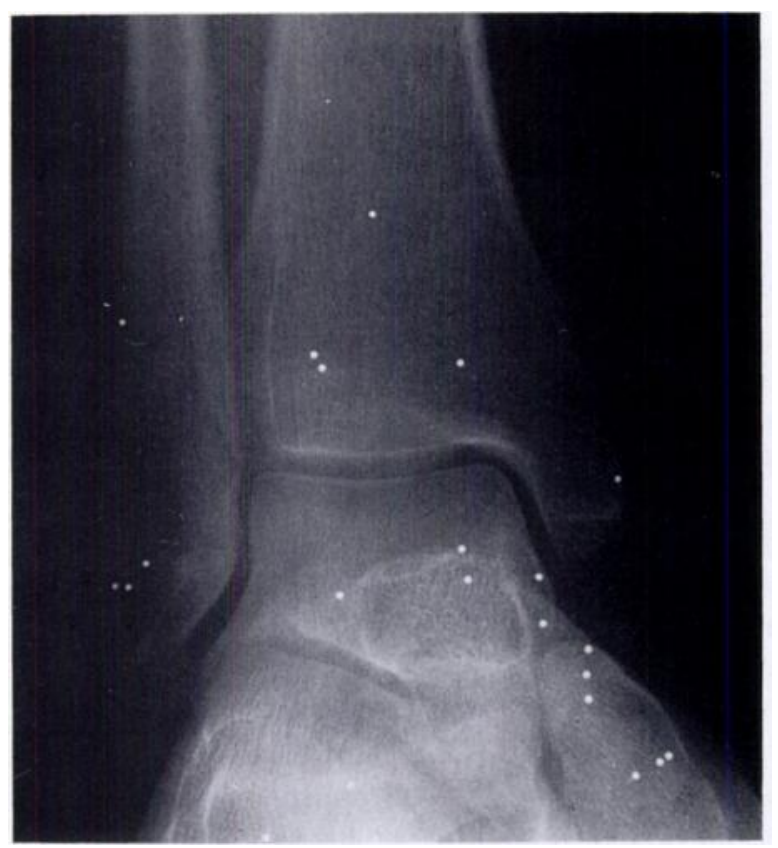

Fig. 2

Anteroposterior view with markers in place. Medial rotation of the platform yields a true anteroposterior view of the mortise and makes easier the identification of the markers in the bones of the longitudinal arch. from the starting point in medial rotation). In this part of the arc the joint axis was found to run downward and medially.

The position of the axis of movement of the ankle joint is important for the calculation of kinetic data, such as joint loads and torques, and for the correct positioning of the axis for an artificial joint. There is, therefore, good reason to study the position of the axis in vivo, using techniques of indisputable accuracy. Roentgen stereophotogrammetry offers such accuracy and can be used to study healthy subjects (Selvik, Alberius and Aronsson 1983; Kärrholm, Hansson and Selvik 1985; Ahl et al. 1987).

\section{MATERIALS AND METHODS}

Eight healthy volunteers participated in this investigation. They had given informed consent and the study was approved by the ethical committee of the Karolinska Hospital. The age of the subjects ranged from 26 to 38 years; six were male and two female (Table I). Three or more $0.8 \mathrm{~mm}$ marker beads of tantalum were introduced into each of the tibia and the talus, as well as the fibula, calcaneus, navicular, medial cuneiform and first metatarsal bones of the right foot. There were no complications.

Two to 10 weeks later, the subjects were placed on a platform that could be tilted in $10^{\circ}$ increments about one transverse and one anteroposterior axis. Rotation could also be recorded using a goniometer placed around the lower leg and fixed to the device (Fig. 1). Two x-ray tubes were placed anterior and medial to the foot on the platform and radiopaque reference grids with cassette holders were placed posterior and lateral to the foot. The platform was medially rotated approximately $20^{\circ}$ in relation to the $\mathrm{x}$-ray tubes to get true anteroposterior and lateral views of the ankle (Fig. 2). Simultaneous anteroposterior and lateral exposures were made at $10^{\circ}$ increments from $30^{\circ}$ of plantarflexion to $30^{\circ}$ of dorsiflexion of the foot, from $20^{\circ}$ of pronation to $20^{\circ}$ of supination and from $20^{\circ}$ of medial rotation to $10^{\circ}$ of lateral rotation of the leg. To avoid undue tension in the calcaneal tendon the positions of dorsiflexion were obtained by bending the knee and inclining the lower leg forward rather than by tilting the platform. All exposures were made with full weight-bearing. When the films were developed, the positions of all marker beads were defined in relation to the reference grid. Three dimensional co-ordinates were calculated for all markers and rigid body kinematic analysis was made (Selvik 1974) providing the helical axes for each pair of consecutive positions.

The co-ordinate system in which the axes were determined had its origin at the geometric centre of the set of marker beads in the tibia; the $x$-axis was perpendicular to the plane of film of the lateral radiograph. Use of this co-ordinate system for presentation could lead to confusion since it has no familiar 


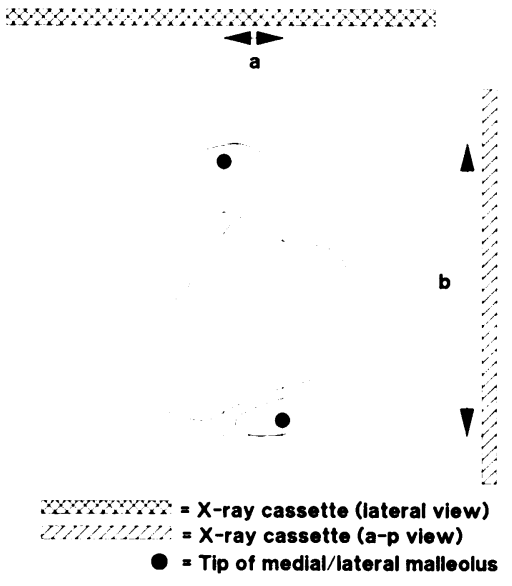

Fig. 3a

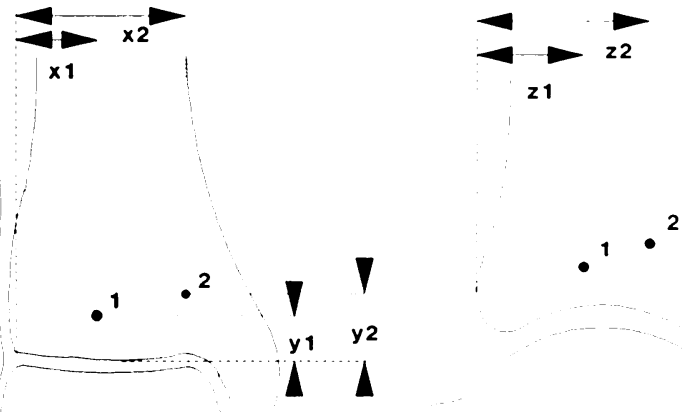

Fig. 3b

Correction of projections. Figure 3a - The distance between projections of the tips of the malleoli are used to calculate the angle between the transmalleolar line and the direction of the rays of the lateral view. This angle can be expressed as arc tan $(a / b)$ where $a$ is the distance between the tips of the malleoli in the lateral view and $b$ is the corresponding distance in the anteroposterior view. Figure $3 \mathrm{~b}$ - The distance of markers from identifiable landmarks was used to calculate the position of the geometric centre of the set of markers in the tibia.
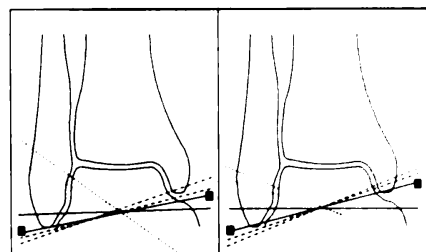

subject 1

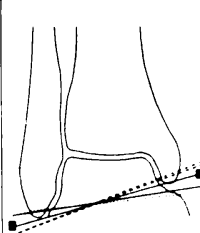

Subject 5
Plantarflexion

30 to $20^{\circ}$

20 to $10^{\circ}$

$10^{\circ}$ to 0

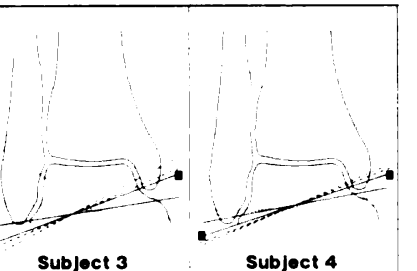

Subjoct 3 Subject 4

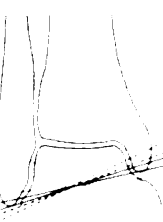

Subject 7

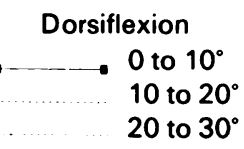

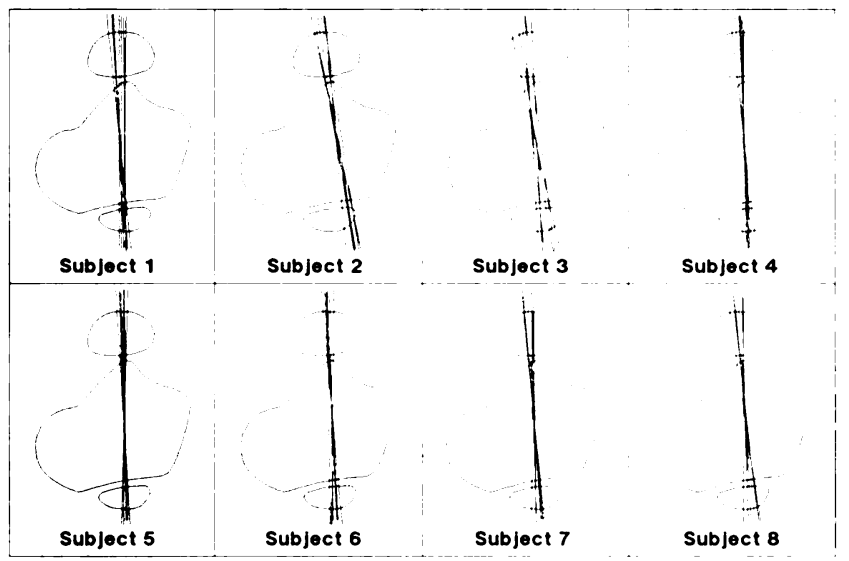

Fig. 5
Individual discrete helical axes projected onto a horizontal plane. Axes tend to fall parallel to a transverse plane through the malleoli.

Fig. 4

Individual discrete helical axes of the talo-crural joint of each subject for each $10^{\circ}$ interval from $30^{\circ}$ of plantarflexion to $30^{\circ}$ of dorsiflexion, projected onto a coronal plane. All plantarflexion axes are more horizontal, or inclining downward and medially, than the dorsiflexion axes. In some subjects the most pronounced plantarflexion axis corresponds to the interval between 20 and $10^{\circ}$ of plantarflexion rather than the interval between 30 and $20^{\circ}$.

anatomical correlation. To avoid this we have presented the results as a co-ordinate system related to the malleoli (Fig. 3).

After correction for differences in width of the ankle joints in different subjects, all joint axes were drawn in a standard set of ankle projections; one coronal, one horizontal and one sagittal view. The angles of inclination in the coronal and sagittal planes were taken directly from the kinematic calculations while the horizontal plane angle was corrected for set-off as mentioned. It should be understood that the correction procedure was used only in order to draw the axes from different individuals in the same illustration; the unknown accuracy of the correction procedure affects only comparison between individuals; the relationships between different axes in each individual are derived directly from the stereophotogrammetric measurements.

\section{RESULTS}

The following patterns were observed:

Dorsiflexion and plantarflexion. From $30^{\circ}$ of plantarflexion to $30^{\circ}$ of dorsiflexion the helical axis of the talo-crural joint, when projected onto a coronal plane, changed position from an inclination which was either downward and medially or horizontal, to a downward and lateral inclination (Fig. 4). Between 10 and $30^{\circ}$ of dorsiflexion 
the helical axis tended to run parallel to a line drawn through the tips of the malleoli. In some subjects there seemed to be two distinct axes, one for plantarflexion and one for dorsiflexion, while in others a more continuous pattern through the whole arc of rotation was seen. The amounts of rotation taking place about the helical axes for each $10^{\circ}$ interval ranged between 3 and $18^{\circ}$ (Tables II and III.)

Projected onto a horizontal plane, the axis always ran close to the tips of the malleoli; any variations in position were insignificant (Fig. 5). When projected onto

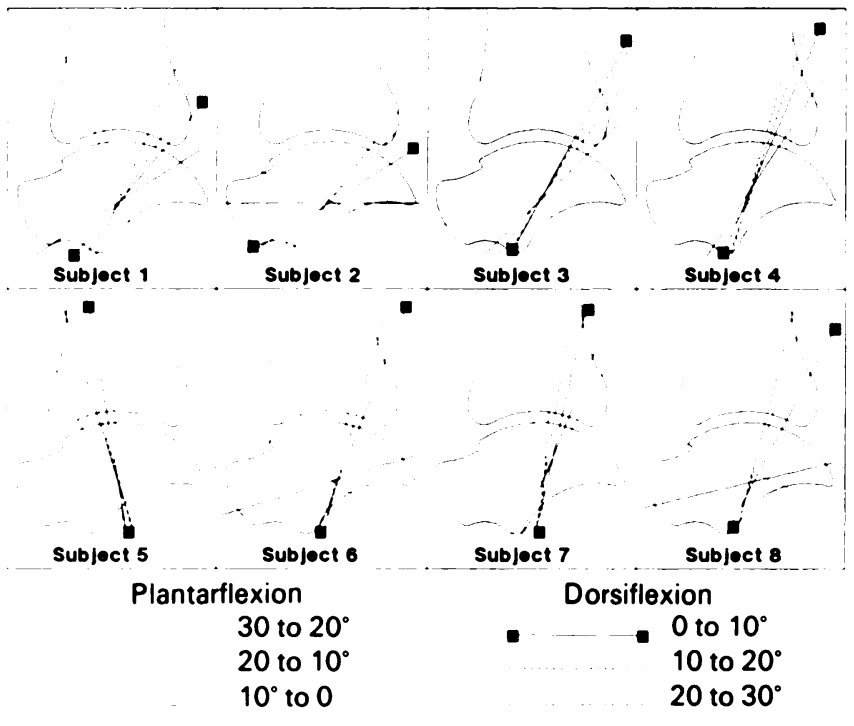

Fig. 6

Individual discrete helical axes projected onto a sagittal plane. Variations in inclination are large because axes are viewed almost endon.

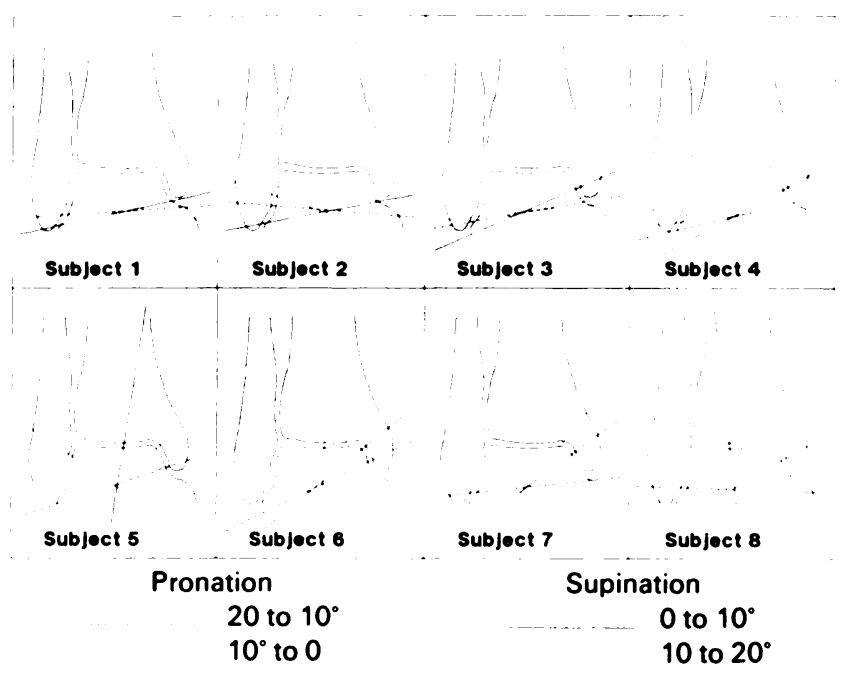

Fig. 7

Individual discrete helical axes of each subject for each $10^{\circ}$ interval from $20^{\circ}$ of pronation to $20^{\circ}$ of supination, projected onto a coronal plane. Only rotations exceeding $1^{\circ}$ are included. a sagittal plane, differences in inclination between the axes were considerable (Fig. 6).

Pronation and supination. From $20^{\circ}$ of pronation to $20^{\circ}$ of supination the average rotations were smaller than in plantarflexion and dorsiflexion (Table IV). Inclinations of the axes in the coronal plane were more variable and values approaching $90^{\circ}$ were occasionally seen (Fig. 7). When projected onto a horizontal plane the axes ran uniformly through the malleoli. In the sagittal plane variation was considerable.

Medial and lateral rotation. Between $20^{\circ}$ of medial rotation of the leg and the neutral position, substantial rotation took place in the talo-crural joint. Inclinations of the axes found in medial and lateral rotation, related to the horizontal plane, ranged from 1 to $88^{\circ}$ (Fig. 8) and the range of rotation was as much as $16^{\circ}$ (Table V). The greatest amounts of rotation were seen in lateral rotation of the leg, occurring about axes inclined at less than $25^{\circ}$ from the horizontal. Projections onto the horizontal and sagittal planes were similar to those seen in dorsiflexion and plantar flexion.

\section{DISCUSSION}

Our investigation fully supports the findings of Barnett and Napier (1952) and Hicks (1953) that the talo-crural joint uses different axes for plantarflexion and dorsiflexion. We found that the change between these axes occurred rather abruptly in some subjects and more gradually in others. The greatest difference in the position of the coronal plane axis between plantarflexion and dorsiflexion, ranged between 18 and $63^{\circ}$ (mean $37^{\circ}$ ). In all cases the axis projected onto the horizontal plane

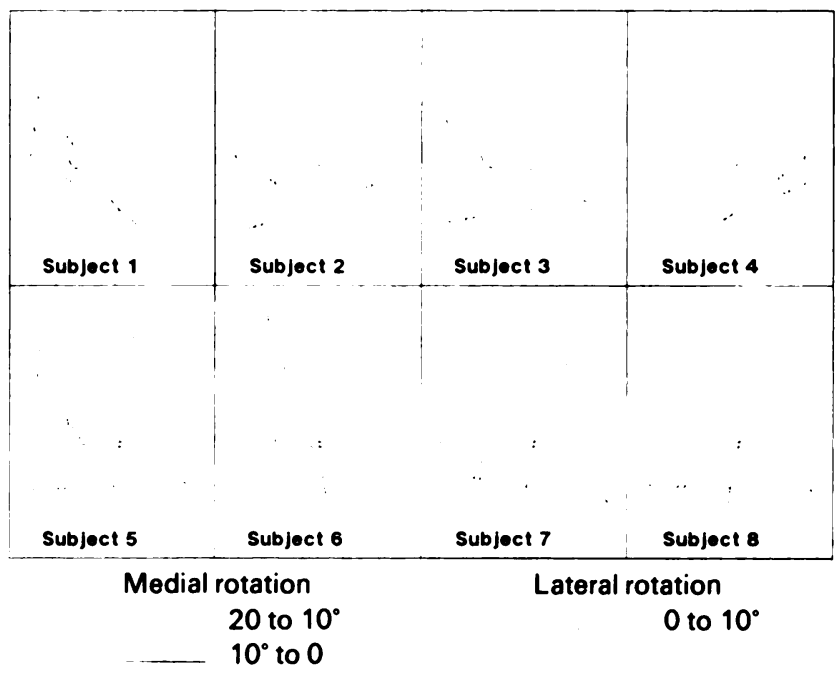

Fig. 8

Individual discrete helical axes of each subject for each $10^{\circ}$ interval from $20^{\circ}$ of medial rotation to $10^{\circ}$ of lateral rotation of the leg, projected onto a coronal plane. Note the steeply vertical course of some axes, particularly in medial rotation. 
Table II. Axes of movement during three intervals of plantarflexion. The axis is given in degrees to the horizontal plane, negative values denoting inclination downward in a medial direction; rotation of the axis is given in degrees for each $10^{\circ}$ interval

\begin{tabular}{|c|c|c|c|c|c|c|}
\hline \multirow[b]{3}{*}{ Subject } & \multicolumn{6}{|c|}{ Range of plantarflexion } \\
\hline & \multicolumn{2}{|c|}{$30-20^{\circ}$} & \multicolumn{2}{|c|}{$20-10^{\circ}$} & \multicolumn{2}{|c|}{$10^{\circ}-0$} \\
\hline & Axis & Rotation & Axis & Rotation & Axis & Rotation \\
\hline 1 & -22 & 4.1 & -34 & 3.3 & 3 & 11.1 \\
\hline 2 & -9 & 4.7 & -10 & 10.1 & 6 & 10.6 \\
\hline 3 & -3 & 3.9 & -37 & 5.1 & 11 & 12.5 \\
\hline 4 & 4 & 3.7 & 9 & 13.7 & 10 & 11.1 \\
\hline 5 & -23 & 13.4 & 8 & 12.9 & 12 & 13.7 \\
\hline 6 & -13 & 9.7 & -6 & 9.2 & 4 & 12.2 \\
\hline 7 & -25 & 4.1 & 9 & 11.8 & 17 & 12.3 \\
\hline 8 & -5 & 15.7 & -5 & 15.8 & 5 & 9.5 \\
\hline
\end{tabular}

Table III. Axes of movement during dorsiflexion, given in relation to the horizontal plane, with rotation of the axis at $10^{\circ}$ intervals

\begin{tabular}{|c|c|c|c|c|c|c|}
\hline \multirow[b]{3}{*}{ Subject } & \multicolumn{6}{|c|}{ Range of dorsiflexion } \\
\hline & \multicolumn{2}{|c|}{$0-10^{\circ}$} & \multicolumn{2}{|c|}{$10-20^{\circ}$} & \multicolumn{2}{|c|}{$20-30^{\circ}$} \\
\hline & Axis & Rotation & Axis & Rotation & Axis & Rotation \\
\hline 1 & 10 & 9.6 & 14 & 8.1 & 23 & 7.4 \\
\hline 2 & 16 & 4.9 & 18 & 10.2 & 21 & 9.3 \\
\hline 3 & 20 & 8.0 & 20 & 6.4 & 26 & 9.9 \\
\hline 4 & 20 & 5.9 & 21 & 10.5 & 22 & 12.0 \\
\hline 5 & 17 & 5.7 & 19 & 8.9 & 20 & 8.9 \\
\hline 6 & 12 & 4.2 & 25 & 9.9 & 25 & 8.4 \\
\hline 7 & 17 & 9.2 & 17 & 17.9 & 26 & 3.2 \\
\hline 8 & 12 & 6.5 & 17 & 9.0 & 15 & 4.8 \\
\hline
\end{tabular}

Table IV. Axes of movement during pronation and supination, related to the horizontal plane. Negative values denote inclination downward in a medial direction. Rotation of the axis is given for $10^{\circ}$ intervals

\begin{tabular}{|c|c|c|c|c|c|c|c|c|}
\hline \multirow[b]{3}{*}{ Subject } & \multicolumn{8}{|c|}{ Range of pronation (P) and supination (S) } \\
\hline & \multicolumn{2}{|c|}{ P 20-10 } & \multicolumn{2}{|c|}{ P $10^{\circ}-0$} & \multicolumn{2}{|c|}{$S 0-10^{\circ}$} & \multicolumn{2}{|c|}{$\mathrm{S} 10-20^{\circ}$} \\
\hline & Axis & Rotation & Axis & Rotation & Axis & Rotation & Axis & Rotation \\
\hline 1 & 3 & 2.7 & -2 & 1.8 & 15 & 3.8 & 75 & 0.7 \\
\hline 2 & -1 & 3.2 & -25 & 0.3 & 12 & 2.9 & -20 & 2.7 \\
\hline 3 & 3 & 2.4 & 11 & 2.6 & 25 & 4.6 & 2 & 1.8 \\
\hline 4 & 13 & 2.8 & 24 & 0.9 & 27 & 2.2 & 5 & 3.6 \\
\hline 5 & 17 & 4.6 & 43 & 1.8 & 80 & 1.5 & -35 & 2.5 \\
\hline 6 & 28 & 1.0 & 16 & 0.7 & 40 & 1.8 & 86 & 2.8 \\
\hline 7 & 2 & 2.6 & 33 & 0.7 & 32 & 2.0 & 50 & 6.6 \\
\hline 8 & -3 & 1.3 & 12 & 2.8 & -83 & 2.2 & 24 & 5.8 \\
\hline
\end{tabular}

Table V. Axes of movement during rotation of the leg on the foot, negative values denoting inclination downward in a medial direction. Rotation of the axis is given for $10^{\circ}$ intervals

\begin{tabular}{|c|c|c|c|c|c|c|}
\hline \multirow[b]{3}{*}{ Subject } & \multicolumn{6}{|c|}{ Range of rotation } \\
\hline & \multicolumn{2}{|c|}{ Medial 20-10 } & \multicolumn{2}{|c|}{ Medial $10^{\circ}-0$} & \multicolumn{2}{|c|}{ Lateral 0-10 } \\
\hline & Axis & Rotation & Axis & Rotation & Axis & Rotation \\
\hline 1 & -34 & 1.8 & -43 & 2.9 & -58 & 3.4 \\
\hline 2 & 84 & 2.8 & -35 & 2.2 & 17 & 8.5 \\
\hline 3 & 84 & 2.4 & -51 & 1.5 & 9 & 8.6 \\
\hline 4 & 83 & 4.6 & 39 & 5.8 & 24 & 14.6 \\
\hline 5 & -51 & 3.7 & 83 & 2.9 & 2 & 2.6 \\
\hline 6 & -71 & 3.1 & -82 & 3.2 & -49 & 1.9 \\
\hline 7 & -23 & 2.2 & 81 & 6.4 & -5 & 4.5 \\
\hline 8 & -88 & 4.5 & 79 & 1.7 & -1 & 4.6 \\
\hline
\end{tabular}

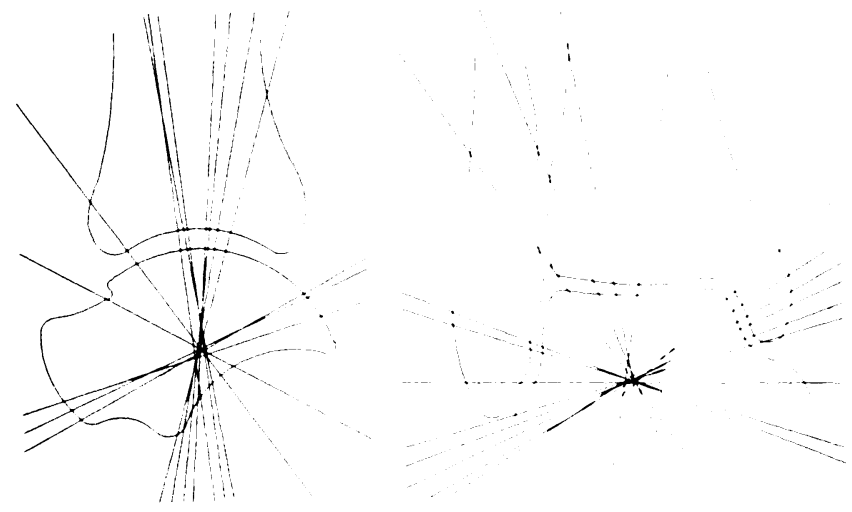

Fig. 9

All coronal and sagittal plane projections of plantarflexion and dorsiflexion, pronation and supination and medial and lateral rotation axes seen in Subject 6 . Note the tendency of axes to cross in one small area within the talus. Similar patterns vere observed in most other subjects. 
ran through both malleoli and close to the centre of both. In the sagittal plane, projection differences in inclination were considerable; this is explained by the axes being seen more or less end-on. There was, on the other hand, considerable consistency in the finding that the axes tended to cross at, or near, one central point in the talus.

The amounts of rotation occurring at the ankle joint as a result of rotation of the leg on the foot were substantial. Values for lateral rotation of the leg were generally higher but the inclination of the axis to the horizontal plane was less pronounced than in medial rotation. The tendency of the joint axis to take a more vertical course in medial rotation was even more pronounced in the present investigation than in van Langelaan's (1983). Close (1956) did not give any information on the position of the joint axis in relation to observed amounts of vertical axis motion, and McCullough and Burge (1980) used an experimental technique that seems to have allowed movement only about the true vertical axis.

It is a significant finding that when plantarflexion and dorsiflexion, pronation and supination and medial and lateral rotational axes for each of our subjects are drawn in the same figure, all axes, irrespective of their inclination, coincide or run very close to one central point in the trochlea of the talus (Fig. 9). This central point seems to constitute a hub, around which the ankle joint has more freedom of movement than is often assumed. The existence of such a centre of movement has several implications, one being that attention should be paid to the position of the centre of the talar part of a spherical total ankle prosthesis. The wide variations in joint axes calls for care in designing models of the ankle. In our investigation, the central point seemed to be located at, or slightly lateral to, the midpoint of a line drawn between the tips of the malleoli.

This study was supported by grants from the Swedish Defence Materials Administration, LIC Orthopaedics and the research funds of the Karolinska Institute.

No benefits in any form have been received or will be received from a commercial party related directly or indirectly to the subject of this article.

\section{REFERENCES}

Ahl T, Dalén N, Lundberg A, Selvik G. Mobility of the ankle mortise. Acta Orthop Scand 1987;58:401-2.

Allard P, Thiry PS, Duhaime M. Estimation of the ligaments' role in maintaining foot stability using a kinematic model. Med Biol Eng Comput 1985;23:237-42.

Barnett CH, Napier JR. Axis of rotation at ankle joint in man: its influence upon the form of talus and mobility of fibula. $J$ Anat 1952:86:1-9.

Close JR. Some applications of the functional anatomy of the ankle joint. J Bone Joint Surg [Am] 1956;38-A :761-81.

Hicks JH. Mechanics of the foot: joints. J Anat 1953;87: $345-57$.

Inman VT. The joints of the ankle. Baltimore: Williams \& Wilkins, 1976.

Kärrholm J, Hansson LI, Selvik G. Mobility of the lateral malleolus: a roentgen stereophotogrammetric analysis. Acta Orthop Scand $1985: 56: 479-83$. van Langelaan EJ. A kinematical analysis of the tarsal joints: an $\mathrm{x}$-ray photogrammetric study. Acta Orthop Scand 1983:54:Suppl 204.

Larsen E. Experimental instability of the ankle: a radiographic investigation. Clin Orthop 1986;204;193-200.

Laurin C, Mathieu J. Sagittal mobility of the normal ankle. Clin Orthop 1975;108:99-104.

McCullough CJ, Burge PD. Rotatory stability of the load-bearing ankle: an experimental study. J Bone Joint Surg [Br] $1980: 62-B: 460-4$

Selvik G. A roentgen stereophotogrammetric method for the study of the kinematics of the skeletal system. Thesis. Lund: AV-centralen, 1974.

Selvik G, Alberius P, Aronson AS. A roentgen stereophotogrammetric system: construction, calibration and technical accuracy. Acto Radiol Diagn 1983;24:343-52.

Stormont DM, Morrey BF, An K, Cass JR. Stability of the loaded ankle: relation between articular restraint and primary and secondary static restraints. AM J Sports Med 1985;23:295-300. 